\title{
Combined modelling of the He2-131 planetary nebula and of its central star
}

\author{
Anabel Arrieta ${ }^{1,2}$, Christophe Morisset ${ }^{3,4}$, D. John Hillier ${ }^{2}$, \\ Grażyna Stasińska ${ }^{5}$ and Leonid N. Georgiev ${ }^{2,3}$
}

${ }^{1}$ Universidad Iberoamericana, México, D.F., México ${ }^{2}$ Department of Physics and Astronomy, University of Pittsburgh, Pittsburgh, PA., USA ${ }^{3}$ Instituto Astronomía, Universidad Nacional Autónoma de México, México, D.F., México ${ }^{4}$ Instituto de Astrofísica de Canarias, La laguna, Tenerife, Spain ${ }^{5}$ Observatoire de Paris, CNRS, Université Paris Diderot, Meudon, France

\begin{abstract}
We present a unified model of the central star and the ionized gas of the planetary nebula He2-131. Preliminary values of the nebular and stellar parameters are given together with those of the chemical composition.
\end{abstract}

Keywords. planetary nebulae: individual (He2-131), stars: fundamental parameters

\section{Introduction and observations}

The parameters of planetary nebulae and their central stars are usually obtained in separate studies and in many cases they are not compatible. In this study we try to improve this situation by constructing a unified model of He2-131 (star and nebula) following the technique pioneered by Morisset \& Georgiev (2009). Here we present our preliminary models (Fig. 1). He2-131 spectra show low ionization nebular lines over a bright stellar continuum. The star shows bright wind features. Variation in some photospheric and wind lines have been reported. We constructed the spectrum of the central star using data obtained with FUSE, IUE, HST-GHRS and HARPS extracted from the databases of these observatories. We used an HST $\mathrm{H} \alpha$ image and the UVES spectra for the nebula.

\section{Modeling}

The stellar spectrum in the $900 \AA-7400 \AA$ region was fitted with a CMFGEN (Hillier \& Miller 1998) model. The computed ionizing radiation from this model was used to calculate a Cloudy_3D Morisset(2006) model of the nebula. The process was iterated until the stellar model and the nebular model reproduce simultaneously the observed spectral and imaging features of both star and nebula in the entire spectral range. Examples of the quality of the stellar fit and the consistency of the nebular diagnostics obtained in this procedure are shown in Fig. 1.

The density distribution of the gas was found to be that of a filled sphere with density increasing toward the edge. Table 1 show the parameters of our current best model of the object. Table 2 presents a comparison between stellar and nebular abundances (in $\log$ of the mass fraction). We have to stress that the mass of the star was assumed rather than derived. The stellar mass, together with the derived $T_{\text {eff }}$ and $\log g$ fix the luminosity of the star and its distance. The gravity used in the stellar model is higher than the required by the wings of $\mathrm{H} \gamma$, but models with lower gravity emit to many hard ionizing photons which to explain the nebular spectrum. We are continuing the exploration of the parameter space in order to resolve this discrepancy. 

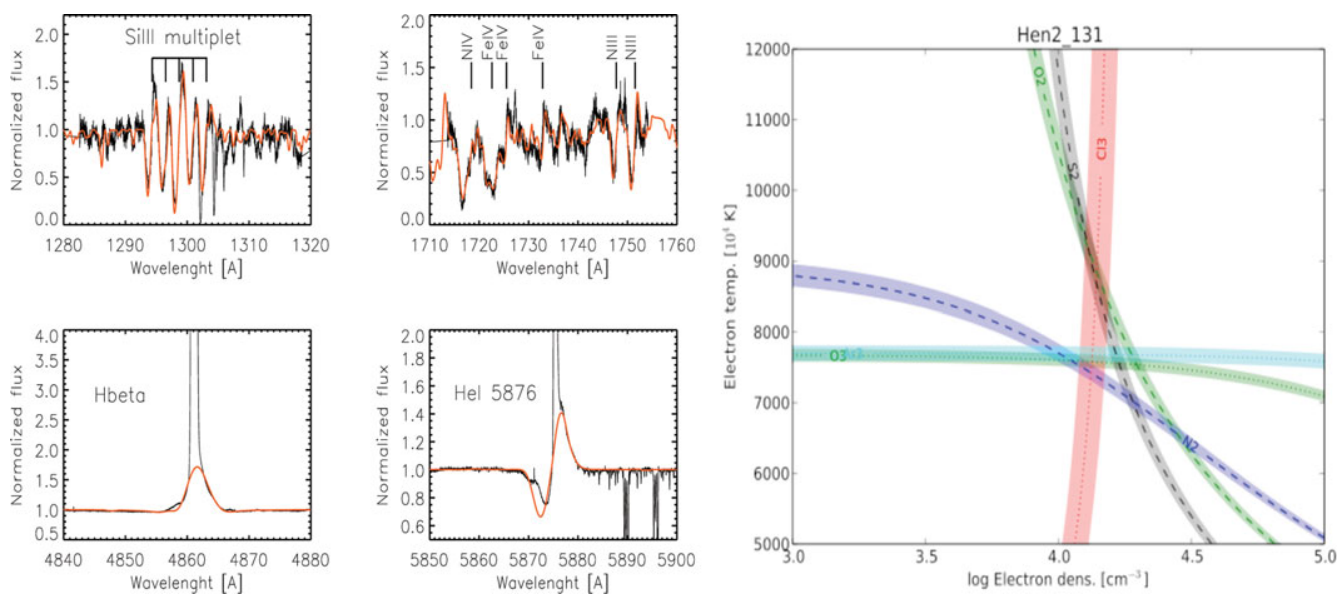

Figure 1. Comparison between observed stellar spectrum and the model (small panels) and nebular diagnostics made with PyNeb (Luridiana et al., these proceedings)(right panel)

Table 1. Parameters of the central star model

\begin{tabular}{lr}
\hline$T_{\text {eff }}$ & $32500 \mathrm{~K}$ \\
$\log L / L \odot$ & 3.77 \\
$\log g$ & 3.5 \\
$V_{\infty}$ & $300 \mathrm{~km} / \mathrm{s}$ \\
$\dot{M}$ & $510^{-8} \mathrm{M} \odot / \mathrm{yr}$ \\
$M_{*} / M_{\odot}($ assumed $)$ & 0.67 \\
$R_{*} / R_{\odot}$ & 13.24 \\
Distance & $2.9 \mathrm{kpc}$ \\
$E(B-V)$ & 0.137 \\
$R$ & 2.5 \\
\hline Electron temp. & $7800 \mathrm{~K}$ \\
Electron density & $210^{4} \mathrm{~cm}^{-3}$ \\
\hline
\end{tabular}

\section{Conclusions}

So far, we have obtained a reasonably consistent model of the planetary nebula He2-131 (central star and nebula), but certain details still need to be worked out. The star shows increased nitrogen abundance which point to a high mass progenitor. The nebula has reduced a silicon abundance which is usually associated with dust depletion although no significant dust emission is observed. A complete analysis of the properties of this object and its evolutionary status will be presented in a forthcoming paper.

Table 2. Comparison of stellar and nebular abundances

\begin{tabular}{cccccccccc}
\hline & $\mathbf{H e}$ & $\mathbf{C}$ & $\mathbf{N}$ & $\mathbf{O}$ & $\mathbf{N e}$ & $\mathbf{A l}$ & $\mathbf{S i}$ & $\mathbf{S}$ & $\mathbf{A r}$ \\
\hline Star & -0.55 & -2.52 & -2.40 & -2.05 & -2.76 & -4.25 & -3.15 & -3.44 & -4.00 \\
Neb. & -0.47 & -2.41 & -2.74 & -2.39 & -2.89 & -4.29 & -4.20 & -3.83 & -4.19 \\
Sun & -0.6 & -2.66 & -3.21 & -2.27 & -2.99 & -4.33 & -3.17 & -3.47 & -2.93 \\
\hline
\end{tabular}

\section{References}

Morisset, C. 2006, IAU Symp. 234, Planetary Nebulae in our Galaxy and Beyond, 467 Morisset, C. \& Georgiev, L. 2009, A\&SA, 507, 1517

Hillier, D. J. \& Miller, D. L. 1998, ApJ, 496, 407 